\title{
A QUICK WAY OF OBTAINING AN APPROXIMATE SOLUTION TO A STURM-LIOUVILLE PROBLEM
}

\author{
LL. G. CHAMBERS
}

ABSTRACT. Use is made of the generalized consistency equation and the boundary conditions to obtain a quick approximate solution to a Sturm-Liouville problem.

\section{INTRODUCTION}

Consider the problem defined by the Sturm-Liouville equation

$$
-\frac{d}{d x}\left\{p(x) \frac{d u}{d x}\right\}+q(x) u=f(x), \quad x_{1} \leq x \leq x_{2},
$$

with $p\left(x_{1}\right), p\left(x_{2}\right)$ nonzero, and boundary conditions of the form

$$
-p\left(x_{1}\right) u^{\prime}\left(x_{1}\right)+r_{1} u\left(x_{1}\right)=g_{1}, \quad p\left(x_{2}\right) u^{\prime}\left(x_{2}\right)+r_{2} u(2)=g_{2} .
$$

Multiplying equation (1.1) by $u(x)$, integrating between $x_{1}$ and $x_{2}$, and using equations (1.2), one obtains

$$
\begin{aligned}
\int_{x_{1}}^{x_{2}}[ & \left.p\left\{u^{\prime}(x)\right\}^{2}+q\{u(x)\}^{2}\right] d x+r_{1}\left\{u\left(x_{1}\right)\right\}^{2}+r_{2}\left\{u\left(x_{2}\right)\right\}^{2} \\
= & \int_{x_{1}}^{x_{2}} f u d x+g_{1} u\left(x_{1}\right)+g_{2} u\left(x_{2}\right) .
\end{aligned}
$$

The condition that the problem defined by (1.1) and (1.2) has a unique solution is equivalent to $u$ being identically zero over $x_{1} \leq x \leq x_{2}$ when the excitations $f(x), g_{1}$, and $g_{2}$ are zero. That is, the quadratic form on the left-hand side of $(1.3)$ is positive definite.

The conditions are as follows: $q, r_{1}$, and $r_{2}$ are nonnegative, and $p$ is positive but may be zero at isolated points in $x_{1}<x<x_{2}$. If $q, r_{1}, r_{2}$ are all zero, however, the problem degenerates to that defined by

$$
\begin{gathered}
-\frac{d}{d x}\left\{p(x) \frac{d u}{d x}\right\}=f(x), \quad x_{1} \leq x \leq x_{2}, \\
-p\left(x_{1}\right) u^{\prime}\left(x_{1}\right)=g_{1}, \quad p\left(x_{2}\right) u^{\prime}\left(x_{2}\right)=g_{2} .
\end{gathered}
$$

Received by the editor July $28,1992$.

1991 Mathematics Subject Classification. Primary 65L10, 34B24.

Key words and phrases. Approximate solution, Sturm-Liouville problem. 
Note that in this case a consistency condition

$$
0=\int_{x_{1}}^{x_{2}} f d x+g_{1}+g_{2}
$$

must hold, and that $u$ is indefinite by an arbitrary constant.

It is equation (1.5) which suggests the possibility of obtaining an approximate solution for the problem defined by (1.1) and (1.2) when a unique solution exists.

\section{Generalized CONSISTENCY EQUATION}

Integrating the differential equation (1.1) over $x_{1} \leq x \leq x_{2}$ and substituting the boundary conditions (1.2), one finds

$$
\int_{x_{1}}^{x_{2}} q u d x+r_{1} u\left(x_{1}\right)+r_{2} u\left(x_{2}\right)=\int_{x_{1}}^{x_{2}} f d x+g_{1}+g_{2} \text {. }
$$

This equation, by virtue of its connection with equation (1.5), will be termed the generalized consistency equation, and a mean value $w_{0}$ of $u$ obeying

$$
w_{0}\left[\int_{x_{1}}^{x_{2}} q d x+r_{1}+r_{2}\right]=\int_{x_{1}}^{x_{2}} f d x+g_{1}+g_{2}
$$

may be defined, the quantity in square brackets being intrinsically positive. Thus, the locality of the solution can be estimated by means of two simple integrations. Equation (2.1) can also be used to obtain an approximate quadratic solution for $u$ :

(2.3) Let $w$ be an approximation to $u$ of the form $w=\alpha+\beta x+\gamma x^{2}$.

Then the set of three equations

(2.4a) $-p\left(x_{1}\right) w^{\prime}\left(x_{1}\right)+r_{1} w\left(x_{1}\right)=g_{1}, \quad p\left(x_{2}\right) w^{\prime}\left(x_{2}\right)+r_{2} w\left(x_{2}\right)=g_{2}$,

$$
\int_{x_{1}}^{x_{2}} q w d x+r_{1} w\left(x_{1}\right)+r_{2} w\left(x_{2}\right)=\int_{x_{1}}^{x_{2}} f d x+g_{1}+g_{2}
$$

makes it possible to determine $\alpha, \beta$, and $\gamma$.

\section{AN EXAMPLE}

Consider the example

$$
\begin{aligned}
& -\frac{d^{2} u}{d x^{2}}+u=1, \quad 0 \leq x \leq 1, \\
& u^{\prime}(0)=0, \quad u^{\prime}(1)+u(1)=0 .
\end{aligned}
$$

The uniqueness conditions are satisfied and the generalized consistency condition is

$$
\int_{0}^{1} u d x+u(1)=1 .
$$

The exact solution of the problem defined by the relations (3.1) is given by

$$
u=1-e^{-1} \cosh x .
$$


The mean value $w_{0}$ of the solution is given by

$$
w_{0}\left[\int_{0}^{1} 1 d x+1\right]=1, \quad \text { whence } w_{0}=.5 \text {. }
$$

The approximate solution

$$
w=\alpha+\beta x+\gamma x^{2}
$$

must obey

$$
w^{\prime}(0)=0, \quad w^{\prime}(1)+w(1)=0, \quad \int_{0}^{1} w d x+w(1)=1
$$

whence

$$
w=\frac{1}{14}\left(9-3 x^{2}\right)
$$

A comparison follows:

$$
\begin{array}{ccc}
x & u(x) & w(x) \\
0 & .6321 & .6429 \\
.5 & .5832 & .5893 \\
1.0 & .4323 & .4286
\end{array}
$$

\section{Comments}

Clearly, it is not easy, and may well be impossible to make an error estimate for the approximate solution obtained by this method. Nevertheless, it is a method which produces an idea of the behavior of the solution and of its approximate values with remarkably little effort. All that is required is evaluations of the integrals $\int_{x_{1}}^{x_{2}} q w d x$ and $\int_{x_{1}}^{x_{2}} f d x$.

In particular, an approximate solution can be produced when $q$ and $f$ are only known numerically. The method will presumably be particularly effective when $p, q$, and $f$ are monotonically increasing or decreasing.

School of Mathematics, University College of North Wales, Bangor, GWynedd LL57 IUT, WALES 